\title{
A modified-Delphi study of a framework to support the potential implementation of pharmacist prescribing.
}

JEBARA, T., CUNNINGHAM, S., MACLURE, K., PALLIVALAPILA, A., AWAISU, A., AL HAIL, M. and STEWART, D. 
A modified-Delphi study of a framework to support the potential implementation of pharmacist prescribing

Tesnime Jebara ${ }^{1}$, Scott Cunningham ${ }^{1}$, Katie MacLure ${ }^{1}$, Abdulrouf Pallivalapila ${ }^{2}$, Ahmed Awaisu $^{3}$, Moza Al Hail $^{2}$, Derek Stewart ${ }^{3}$

${ }^{1}$ School of Pharmacy and Life Sciences, Robert Gordon University, Aberdeen, United Kingdom, AB10 7GJ

${ }^{2}$ Pharmacy Department, Women's Wellness and Research Center, Hamad Medical Corporation, Doha,

Qatar, PO Box 3050

${ }^{3}$ College of Pharmacy, Qatar University Health, Qatar University, Doha, Qatar, P.O. Box 2713

Correspondence to:

Professor Derek Stewart

College of Pharmacy

Health Cluster

Qatar University

Doha

Qatar

Tel: (+974) 44035562

d.stewart@qu.edu.qa

The authors confirm that the Principal Investigator for this paper is Dr Tesnime Jebara.

Declaration of interest: None.

Sources of funding: This research did not receive any specific grant from funding agencies in the public, commercial, or not-for-profit sectors. 


\section{ABSTRACT}

Background: There is an extensive evidence base of the effectiveness and safety of pharmacist prescribing around the globe. There is therefore potential to develop a framework to support the implementation in Qatar to achieve its National Vision 2030 of establishing a comprehensive world-class healthcare system by better utilisation of its healthcare workforce.

Aim: To determine the levels of agreement amongst key stakeholders regarding a framework for the potential development and implementation of pharmacist prescribing in Qatar.

Method: A quantitative, consensus-based modified Delphi study involving stakeholders in Qatar with key strategic positions of health policy influence (directors of medicine/nursing/pharmacy, lead administrators, health-related academics, patient safety leads, professional regulators) was conducted.

Delphi statements were developed from extensive literature reviews, semi-structured interviews, pharmacist prescribing frameworks implemented in other countries, and based on the Consolidated Framework for Implementation Research. The scope of the statements included definitions and scope of prescribing, education and training, and governance, were validated with eight specialists from UK and Qatar, and presented as an online Delphi. Consensus was set at $70 \%$ or higher agreement and less than $15 \%$ disagreement for each statement.

Results: Thirty-three experts agreed to participate in the Delphi, with a response rate of $94 \%$ for Round 1 and $91 \%$ for Round 2, at which point the Delphi stopped. Consensus was achieved for 38 out of 47 statements indicating that a collaborative prescribing model was preferred, and that experience and additional training were required along with robust governance. Consensus was not reached in relation to independent prescribing, prescribing controlled drugs, and ordering certain diagnostic/monitoring investigations (e.g. ECG, X-ray).

Conclusion: High levels of agreement were attained for statements, which can constitute a framework for the development and implementation of pharmacist prescribing in Qatar. Further work is required to translate this framework into healthcare policy and practice.

Keywords: pharmacist prescribing, pharmacy, framework, implementation, Qatar 


\section{Introduction}

It is estimated that one in 10 patients continues to be harmed whilst receiving healthcare [1]. Many studies have highlighted the prevalence of medication errors and their associated causes, with most literature focusing on errors in prescribing [2-6]. Prescribing can be a challenging activity, with many different and sometimes conflicting issues to consider, as highlighted in the 'Principles of Good Prescribing' articulated by the British Pharmacological Society in 2010 (Table 1) [7].

\section{Table 1: Principles of good prescribing [7]}

- Be clear about the reasons for prescribing

- $\quad$ Take into account the patient's medication history before prescribing

- Take into account other factors that might alter the benefits and risks of treatment

- Take into account the patient's ideas, concerns and expectations

- Select effective, safe and cost-effective medicines individualised for the patient

- Adhere to national guidelines and local formularies were appropriate

- Write unambiguous legal prescriptions using the correct documentation

- Monitor the beneficial and adverse effects of medicines

- Communicate and document prescribing decisions and the reasons for them

- Prescribe within the limitations of your knowledge, skills and experience

In 2017, the World Health Organization (WHO) published the third Global Safety Challenge, 'Medication Without Harm' with the aim of reducing errors by $50 \%$ by 2022 . Countries and organisations are strongly encouraged to review medication-related structures and processes and to develop action plans to meet this aim within the timeframe [8].

Pharmacists have an extensive medication-related education and training hence can contribute to optimal prescribing and appropriate use of medications. Many countries, including the United Kingdom (UK), the United States (US), Canada, and New Zealand have introduced legislation to implement pharmacist prescribing, though the definitions, training and scope of practice vary considerably [9]. Pharmacist prescribing is most advanced in the UK [10] with the implementation of supplementary prescribing in 2003 and independent prescribing in 2006 [11-13]. Independent prescribing, defined as 'prescribing by a practitioner (e.g. doctor, dentist, nurse, pharmacist) responsible and accountable for the assessment of patients with undiagnosed or diagnosed conditions and for decisions about the clinical management required, including prescribing', allows suitably trained pharmacists to prescribe within their competence the same range of medications as physicians [14]. 
Pharmacist prescribing is described within the framework of non-medical prescribing, with aims of: improving patient care outcomes without compromising safety; making it easier for patients to access medications; increasing patient choice; making better use of the skills of health professionals; and contributing to the introduction of more flexible team working across the National Health Service [14]. Education, training, models of practice and related governance related to pharmacist prescribing are all clearly defined [15-16]. Furthermore, in 2012, a competency framework for all prescribers in the UK including pharmacists was introduced, which was subsequently updated in 2016, setting out the skills and characteristics prescribers should possess in order to ensure effective performance [15]. The aims and frameworks of pharmacist prescribing clearly align to the aspirations of the WHO, for improving both patient care and safety.

Several systematic reviews have reported different aspects of pharmacist prescribing practice. A Cochrane review of 20 studies of pharmacist prescribing and 26 of nurse prescribing reported that practising with varying but high levels of prescribing autonomy in a range of settings and for acute and chronic conditions, pharmacist and nurse prescribers were as effective as medical prescribers [17]. For instance, comparable outcomes were achieved for systolic blood pressure, glycated haemoglobin, low-density lipoprotein cholesterol, medication adherence, patient satisfaction, and health-related quality of life [17]. A more recent systematic review of 65 studies of the views and experiences of key stakeholders on pharmacist prescribing reported an accumulation of positive evidence. The benefits reported by studies included: ease of patient access to healthcare; improved patient outcomes; better use of pharmacists' skills and knowledge; improved pharmacist job satisfaction; and reduced physician workload [9]. There is also emerging evidence of objective measures of safe prescribing. A study of pharmacist prescribing in three hospitals in England reported an error prevalence of $0.3 \%$ [18], much lower than that reported in systematic reviews of physician prescribing errors $[2,19]$. A pilot study of pharmacist prescribers' performance on the UK Prescribing Safety Assessment showed that pharmacists performed equivalently to final year medical students in all assessment areas, with a slightly lower performance in the prescribing, drug monitoring and data interpretation questions offset by better performance in prescription review and adverse drug reactions [20]. This body of evidence can support development and implementation of pharmacist prescribing in other countries. However, most studies conducted to date have originated from the Western countries. This, together with the varying definitions and models of pharmacist prescribing may limit the applicability of 
these models elsewhere, particularly if there are major differences in culture, healthcare system, policies, laws and regulations.

Over the past decade, Qatar has transformed its healthcare system to achieve better patient outcomes as outlined in the Qatar National Vision 2030 [21]. This aspires to establish, 'a comprehensive world-class healthcare system whose services are accessible to the whole population' and create 'an integrated system of healthcare offering high-quality services through public and private institutions' with a focus on shifting delivery of care towards primary settings. Improving disease management by ensuring early diagnosis, effective treatment, research, and utilising its skilled and motivated workforce is also high on the agenda [22-23]. The development and implementation of models of pharmacist prescribing in Qatar may therefore go some way to achieving these aims and aspiration by helping to effectively manage patients closer to home thus improving their clinical and humanistic outcomes as well as reducing reliance on secondary care settings by better utilising the knowledge and skills of pharmacists. A recent qualitative study explored the views and perceptions of key stakeholders with strategic influence in Qatar regarding pharmacist prescribing. Findings demonstrated support, with preference for a more conservative model initially and emphasis on education, training, and governance [24].

The aim of this study was to determine the levels of agreement amongst key stakeholders in Qatar regarding a framework for the potential development and implementation of pharmacist prescribing.

\section{Methods}

\section{Study design}

The design was a quantitative, consensus-based, modified-Delphi study with key health-related stakeholders in Qatar. Consensus approaches are particularly useful in developing policies, supporting governance, and stimulating debate around areas where there is uncertainty or incomplete evidence [25-28]. Delphi is an approach to obtain the most reliable consensus in a systematic manner by employing a series of well-defined questionnaires [28]. This study was a modified-Delphi [27, 29] as the questionnaire was based on review of the literature [9-10] and prior qualitative interviews [24].

\section{Setting}

Data collection took place in Qatar, across all major healthcare institutions relating to the education, practice, regulation and governance of pharmacists. These included the Ministry of Public Health, primary, 
secondary, and tertiary healthcare settings, and all health academic institutions providing medical, nursing, pharmacy, and pharmacy technician education programmes.

\section{Delphi expert panel inclusion and exclusion criteria}

The main inclusion criteria for the Delphi expert panel was all key stakeholders in their respective fields holding positions of policy influence to facilitate enactment of the research findings. The key groups targeted represented health professionals at Director level (or equivalent) involved in prescribing (medicine, pharmacy and nursing), policy makers, leading administrators, senior educators and regulators, and managers of patient safety and quality assurance. Members of the research team based in Qatar were excluded from the study.

\section{Sampling}

The names and contact details of all experts and key stakeholders in Qatar that fit the inclusion criteria were collected by the local members of the research team using their professional networks. The sampling strata were leaders and directors representing (a) medicine, (b) nursing, (c) pharmacy and pharmacy technicians, (d) policy, (e) quality and patient safety, and (f) academia. At least five experts from each group were randomly selected and invited to participate via email with a reminder sent every two weeks.

\section{Sample size determination}

There is no evidence to guide the size of a Delphi panel [29-30]. The aim was to recruit at least five stakeholders from each of the sampling strata categories giving an estimated panel size of 35 .

\section{Development of data collection tools}

The Delphi statements were developed based on an umbrella review of systematic reviews [10], the systematic review of views on pharmacist prescribing [9], recent qualitative interviews in Qatar [24], and pharmacist prescribing frameworks from other countries [15, 31-34]. The Consolidated Framework for Implementation Research (CFIR) provided the theoretical grounding for the statements [35]. Using a theoretical framework is more likely to provide comprehensive coverage of all key factors compared to a more pragmatic approach [36-37]. CFIR was considered most appropriate for this study as it is a metatheoretical implementation framework developed from 19 related implementation theories and models mainly in the healthcare sector. It is described in five domains of intervention characteristics, outer setting, inner setting, characteristics of individuals, and planning [35].

The content of the Delphi prescribing framework focused on the definitions and scope of prescribing, prescribing models, education and training, competence, accreditation and governance. All draft statements 
were mapped to the five CFIR domains to ensure that all aspects implementation were considered. A definition of each of the different pharmacist prescribing models was also included to ensure that all panel experts have a uniform understanding of the differences between them before attempting to rate the statements. Following the development of the draft statements, eight specialists on pharmacist prescribing and use of the Delphi in UK and Qatar were invited to review the statements for face and content validity. Forty-seven statements were finalised, grouped in three categories of: (a) Definitions, Models, and Scope, (b) Education and Training, and (c) Prescribing Practice and Governance. The statements were formatted in Survey Monkey ${ }^{\circledR}$ (Survey Monkey Inc., San Mateo, California, USA). For each statement, a six-point rating scale was used (strongly disagree — disagree — somewhat disagree - somewhat agree — agree — strongly agree). For each statement, the experts could add comments to justify their response, suggest alterations to the statements, or add any new statements.

\section{Data collection}

Data collection took place from June to July 2018, as summarised in Figure 1.

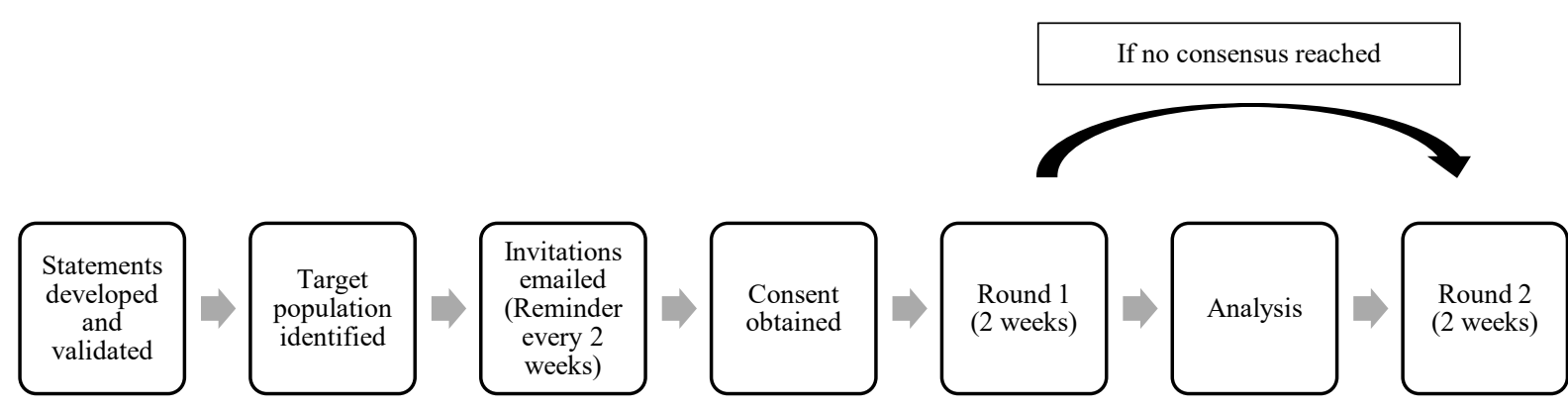

Fig 1: Study flowchart

\section{Data analysis}

While there was no evidence on the most appropriate approach to measuring evidence, it is most frequently referred to as the 'certain level of agreement' [38]. In this study, consensus was deemed as being achieved for statements with more than $70 \%$ agreement (sum of agree and strongly agree) and less than $15 \%$ disagreement (sum of disagree and strongly disagree). Open comments were independently analysed using a summative content analysis (TJ, DS) [39].

\section{Ethics}

Ethical approval was granted by Robert Gordon University School of Pharmacy and Life Sciences Research Ethics Committee (Approval reference S104), Hamad Medical Corporation (HMC) Medical Research Committee (Approval reference MRC-01-17-115), and Qatar University Institutional Review Board 
(Approval reference QU-IRB 865-E/17). Written informed consent was collected from all participants prior to partaking in this study.

Results

\section{Delphi expert panel}

Thirty-three experts agreed to participate, representing all sampling strata and settings, as highlighted in

Table 2.

Table 2: Characteristics of included experts according to practice setting

\begin{tabular}{|c|c|}
\hline Expert category & Setting: Number of experts* \\
\hline Academic Leaders & $\begin{array}{l}\text { - } \text { Medicine: } 1 \\
\text { - } \quad \text { Pharmacy and pharmacy technician: } 3 \\
\text { - Nursing: } 1\end{array}$ \\
\hline Healthcare Policy Developers & $\begin{array}{ll}\text { - } & \text { Secondary care: } 1 \\
\text { - } & \text { Tertiary care: } 1 \\
\text { - } & \text { Corporate/Ministry: } 6\end{array}$ \\
\hline Medical Practice Leaders & $\begin{array}{ll}\text { - } & \text { Primary care/Community: } 1 \\
\text { - } & \text { Secondary care: } 1 \\
\text { - } & \text { Tertiary care: } 1 \\
\text { - } & \text { Corporate: } 4\end{array}$ \\
\hline Pharmacy Practice Leaders & $\begin{array}{ll}\text { - } & \text { Primary care/Community: } 4 \\
\text { - } & \text { Secondary care: } 1 \\
\text { - } & \text { Tertiary care: } 4 \\
\text { - } & \text { Corporate: } 1\end{array}$ \\
\hline Nursing Practice Leaders & $\begin{array}{ll}\text { - } & \text { Secondary care: } 2 \\
\text { - } & \text { Tertiary care: } 4 \\
\text { - } & \text { Corporate/Ministry: } 2 \\
\text { - } & \text { Corporate: } 1\end{array}$ \\
\hline Patient Safety Advocates & $\begin{array}{ll}\text { - } & \text { Primary care/Community: } 1 \\
\text { - } & \text { Tertiary care: } 2 \\
\text { - } & \text { Corporate/Ministry: } 1\end{array}$ \\
\hline
\end{tabular}

*Some experts had multiple roles, e.g. Medical Practice Leader and Healthcare Policy Developer Level of consensus

Thirty-one responses (94\%) were received in Round 1, with consensus achieved for 32/47 statements. The 15 statements that did not reach consensus during Round 1 were either revised $(n=4)$ or left unchanged $(n=11)$ based on the experts' comments. In Round 2, 30 responses (91\%) were received on these 15 
statements and consensus was achieved for a further six statements ( 3 originally unaltered and 3 revised statements). The Delphi statements that reached consensus and those that did not are summarised in Tables 3 and 4. 


\section{Table 3: Summary of final Delphi statements that reached consensus at the end of Round 2}

Statements

Level of consensus

$\%$ Disagreement"

\section{$\%$ Agreement**}

1. Definitions, models and scope

1.1. Pharmacist Collaborative Prescribing

A collaborative model of pharmacist prescribing is appropriate for Qatar. 3.3

The protocol for collaborative pharmacist prescribing should have a defined generic format approved by Qatar Council for 3.2

Healthcare Professionals (QCHP).

The protocol for collaborative pharmacist prescribing must state the targeted medical condition(s). 3.2

The protocol for collaborative pharmacist prescribing must state the scope of prescribing for the pharmacists (e.g. what, when, and 0

how to initiate/continue/discontinue/change drugs, dose, duration...).

The protocol for collaborative pharmacist prescribing must be approved by the pharmacist prescriber(s), physician(s), and the 3.2

pharmacy director within the organisation.

Under the Collaborative Pharmacist Prescribing model, pharmacists can prescribe, within their competence, any over-the-counter 6.5

6.5

drug stated in the protocol.

Under the Collaborative Pharmacist Prescribing model, pharmacists can prescribe, within their competence, any prescription-only 0

drug stated in the protocol.

\subsection{Pharmacist Independent Prescribing for medical conditions previously diagnosed by a physician}

Under the Pharmacist Independent Prescribing for previously diagnosed conditions model, a physician must diagnose the medical 0

condition before the pharmacist can prescribe.

Under the Pharmacist Independent Prescribing for previously diagnosed conditions model, pharmacists can prescribe, within their

12.9

93.5

competence, any over-the-counter drugs. 
1.3. Pharmacist Independent Prescribing for medical conditions which have not been previously diagnosed by a physician

Under the Pharmacist Independent Prescribing for undiagnosed conditions model, pharmacists can prescribe, within their 6.7

competence, any over-the-counter drugs.

\section{Education \& Training}

All pharmacist prescribers must complete a university-led education and training programme accredited by QCHP. 03.5

The education and training programme must be related to the medical condition(s) in which the pharmacist is planning to prescribe. 3.2

The education and training programme must include a period of learning in practice (i.e training) relating to the medical 3.2

87.1

condition(s) in which the pharmacist is planning to prescribe.

The period of learning in practice must be supervised by a senior physician with a particular interest in prescribing. 70

The senior physician supervising the period of learning in practice must be familiar with the education and training programme's $6.5 \quad 90.3$ aims and objectives.

All pharmacists enrolling in the education and training programme must have a postgraduate qualification in clinical pharmacy or a 6.7 related field.

All pharmacists enrolling in the education and training programme must have at least 2 years of direct clinical patient care

experience in the medical condition(s) in which they are planning to prescribe.

All pharmacists enrolling in the education and training programme must have their application endorsed by the senior physician

responsible for the period of learning in practice.

All pharmacists enrolling for the education and training programme must have the endorsement of the pharmacy director in their

organisation.

All pharmacists planning to enrol in the education and training programme must submit a portfolio outlining the set of skills and 0

experience they possess and how they plan to develop further.

All pharmacists enrolling in the education and training programme must demonstrate that, within their area of practice, there is a

clinical need for their prescribing role. 
practice.

\section{Prescribing Practice \& Governance}

Pharmacist prescribers must not commence prescribing practice until registered with QCHP. 03.5

All newly registered pharmacist prescribers must first practise collaborative prescribing for a period of time prior to progressing to 9.7

independent prescribing.

The job description of the pharmacist prescribers must be amended to include prescribing. 6.5

If in doubt about their ability to prescribe for a patient, the pharmacist prescriber must refer him/her back to the physician. 00

All pharmacist prescribers must prescribe according to local policies, guidelines and protocols of their organisation. 00

Once registered, all pharmacist prescribers must undertake Continuing Professional Development (CPD) within the medical 100

condition(s) in which they are prescribing.

All pharmacist prescribers must have ready access to patient clinical records. 03.5

All pharmacist prescribers must document every prescribing activity in the patient clinical records. 00

All pharmacist prescribers must have the authority to order appropriate laboratory tests to inform their prescribing decisions. 3.2

All pharmacist prescribers must report prescribing errors according to the policy of their organisation. 00

All pharmacist prescribers must report adverse drug reactions (ADRs) according to the policy of their organisation. 00

Pharmacists' prescribing practice must be audited regularly against set and accepted standards. 06.8

Patients' feedback on the prescribing practice must be collected regularly, using standardised tools. 07.1

A state-wide campaign should be launched to educate the general public about pharmacist prescribing.

$0 \quad 83.9$

A state-wide campaign should be launched to educate healthcare providers about pharmacist prescribing.

06.8

$* \%$ Disagreement $=\%$ Strongly Disagree $+\%$ Disagree $\quad * * \%$ Agreement $=\%$ Strongly Agree $+\%$ Agree 


\section{Table 4: Summary of final Delphi statements that did not reach consensus at the end of Round 2}

Statements

1. Definitions, models and scope

1.1. Pharmacist Collaborative Prescribing

Under the Collaborative Pharmacist Prescribing model, pharmacists can prescribe, within their competence, any controlled drug

20

50

stated in the protocol.

\subsection{Pharmacist Independent Prescribing for medical conditions previously diagnosed by a physician}

Pharmacist Independent Prescribing for previously diagnosed medical conditions is an appropriate model for Qatar.

Under the Pharmacist Independent Prescribing for previously diagnosed conditions model, pharmacists can prescribe, within their 30

16.7 40 competence, any prescription-only drugs

Under the Pharmacist Independent Prescribing for previously diagnosed conditions model, pharmacists can prescribe, within their

competence, any controlled drugs.

\subsection{Pharmacist Independent Prescribing for medical conditions which have not been previously diagnosed by a physician}

Pharmacist Independent Prescribing for undiagnosed medical conditions is an appropriate model for Qatar. 76.7

Under the Pharmacist Independent Prescribing for undiagnosed conditions model, pharmacists can prescribe, within their

competence, any prescription-only drugs.

Under the Pharmacist Independent Prescribing for undiagnosed conditions model, pharmacists can prescribe, within their

competence, any controlled drugs.

\section{Prescribing Practice \& Governance}

All pharmacist prescribers must have the authority to order other relevant tests and investigations (e.g. ECG, X-ray...) if clinically

indicated.

All pharmacist prescribers must NOT have any role in the dispensing process for the patients for whom they prescribe.

13.3

60

$* \%$ Disagreement $=\%$ Strongly Disagree $+\%$ Disagree

$* * \%$ Agreement $=\%$ Strongly Agree $+\%$ Agree 
Based on the levels of agreement and the comments received in Rounds 1 and 2, it seemed unlikely that consensus would be achieved for the remaining statements in a further round, hence data collection ceased. Content analysis of comments received in the two rounds identified several key issues that led to not reaching consensus on the remaining statements.

An independent prescribing model was not considered appropriate for Qatar at this point in time,

"Qatar is not ready for this approach yet - but yes in the future when the processes have matured and there is greater acceptance from physicians."

Pharmacist prescribing of controlled drugs was contrary to the pharmacy and medicine laws in Qatar,

"The medicolegal situation and the lack of current training and experience makes this a dangerous step."

While there was consensus for pharmacist ordering appropriate laboratory tests to inform their prescribing decisions, this was not extended to more diagnostic tests such as X-rays,

"Who decides what is clinically indicated? Again, we risk blurring the lines about responsibilities of different individuals in the team and fragmenting care."

While permitting prescribing and dispensing for the same patient by the same pharmacist did not reach consensus, there were concerns that this could cause inconvenience for patients in pharmacies with only one pharmacist,

"What is the point in having a pharmacist prescribe and then having a patient go to a different pharmacy to get the medication dispensed, if no other pharmacist is on staff?"

\section{Discussion}

\section{Statement of key results}

Of the 47 statements, consensus was achieved for 38, with high levels of agreement for a pharmacist collaborative prescribing model. There was overwhelming agreement that pharmacist prescribers must undertake a specific, accredited education and training programme prior to prescribing, structured similarly to that of UK independent prescribing with university and practice-based components [16]. Pharmacist prescribers 
must practise within their competence and a framework of governance, aligned to the standards of Qatar Council for Healthcare Professionals (QCHP).

\section{Interpretation of findings}

Consensus approaches are generally considered to generate a relatively low level of evidence [40], but particularly useful in the development of frameworks, guidance and policy statements [26, 28]. Others have used consensus methods for aspects of non-medical prescribing including service redesign around pharmacist prescribing in the UK hospital setting [41], and defining those medical conditions which could be managed by community practitioner nurse prescribers [42].

While systematic reviews of the effectiveness and acceptability of pharmacist prescribing have generated positive findings, the authors acknowledged the variations in the definitions and specific models of prescribing [10]. In this study, consensus was achieved only for the collaborative prescribing model. Collaborative prescribing in the US requires documentation of potential prescribing options, allowing commencement and alteration of medicines and assessing patients' response to treatment. The prescribing plan is agreed between one or more physicians and the pharmacist and can be applied to a group of patients who fulfil the criteria documented in the plan [32]. Collaborative prescribing is therefore likely to be less bureaucratic and more acceptable than supplementary prescribing in the UK which requires an individual patient clinical management plan, agreed by the physician, supplementary prescriber and patient prior to prescribing [43-44].

While there was no agreement to implement independent prescribing from the outset, citing the immaturity of the healthcare system generally and pharmacy practice, it was acknowledged that this could be considered in due course. This is similar to the pharmacist prescribing approach in the UK with supplementary prescribing initiated three years prior to independent prescribing [13]. While concerns relating to independent prescribing were also highlighted in a systematic review of stakeholders' views and experiences of pharmacist prescribing in pre-implementation studies, they were not identified post-implementation highlighting the value of the lived experiences of pharmacist prescribing [9].

It is reassuring that there was expert consensus around the need for targeted education and training of pharmacist prescribers in Qatar. Structuring and benchmarking this around the UK model of university training 
combined with a period of learning in practice under the supervision of a trained medical practitioner [16] will provide confidence in the likely effectiveness and safety of prescribing. The role of the regulator in Qatar (QCHP) in accrediting education and training programmes and setting standards for collaborative protocols highlighted in this Delphi study is likely to provide reassurance to all stakeholders that pharmacist prescribing will be safe and effective. Furthermore, the high levels of agreement around the governance of prescribing at the level of the prescriber (e.g. evidence-based prescribing, robust documentation) will also promote quality prescribing.

Initially in the UK, controlled drugs could only be prescribed as part of a supplementary prescribing clinical management plan prior to legislation being altered to allow independent prescribing $[14,45]$. The lack of agreement for pharmacist prescribing of controlled drugs in Qatar is also explained by the legislation in Qatar which mandates that controlled drugs can only be prescribed by a licensed physician [46].

The other issue which was contentious in this Delphi was the need to separate prescribing and dispensing by the same pharmacist with experts highlighting that, in those pharmacies with only one pharmacist, this may negate enhanced patient access to medicines. Conversely, in other countries where pharmacist prescribing was implemented such as the UK and New Zealand, this separation is considered essential to optimise patient safety [33-34, 47]. However, developments such as dispensing automation and development of the pharmacy workforce to include dispensing accuracy technicians may alleviate the concerns expressed by experts in Qatar.

\section{Strengths and limitations}

There are several strengths to this study including the robust approach to statement development which included comprehensive literature reviews [9-10], qualitative interviews [24], and grounding the statements in CFIR [35]. Application of a theoretical framework allowed more comprehensive coverage of issues likely to affect implementation compared to a more pragmatic approach [48]. Further strengths were the participation of a diverse group of stakeholders with strategic positions in Qatar and the high response rates in both Delphi rounds. While data were collected in Qatar and findings may lack generalisability to other countries, it is likely that statements relating to education and training, prescribing practice and governance may be relevant to other healthcare professions other countries. 


\section{Further work}

Further work should now focus on translating this framework into action through developing the curriculum for pharmacist collaborative prescribing and all related policy and governance standards for education, training and practice.

\section{Conclusion}

This study identified high levels of agreement thus consensus from key decision makers and leaders in Qatar on a framework to support the development and implementation of pharmacist prescribing. Further work is now required to translate this framework into healthcare policy and practice which has the potential to improve prescribing practice and patient safety thus aligning to the key aspirations of Qatar and the WHO.

Declaration of interest: None.

Sources of funding: This research did not receive any specific grant from funding agencies in the public, commercial, or not-for-profit sectors. 


\section{References}

1. World Health Organization. 10 facts on patient safety. NK: World Health Organization; 2018. Available from: https://www.who.int/features/factfiles/patient_safety/en/ [Accessed 23/03/2019].

2. Ross $\mathrm{S}$, Bond $\mathrm{C}$, Rothnie $\mathrm{H}$, et al. What is the scale of prescribing errors committed by junior doctors? A systematic review. British Journal of Clinical Pharmacology 2009; 67(6): 629-640.

3. Alanazi M, Tully M, Lewis P. A systematic review of the prevalence and incidence of prescribing errors with high-risk medicines in hospitals. Journal of Clinical Pharmacy and Therapeutics 2016; 41: 239-245.

4. Salmasi S, Wimmer BC, Khan TM, et al. Quantitative exploration of medication errors among older people: a systematic review. Drugs \& Therapy Perspectives 2017; 34(3): 129-137.

5. Assiri GA, Shebl NA, Mahmoud MA, et al. What is the epidemiology of medication errors, error-related adverse events and risk factors for errors in adults managed in community care contexts? A systematic review of the international literature. BMJ Open 2018; 8(5): e019101.

6. Mekonnen AB, Alhawassi TM, Mclachlan AJ, Brien JE. Adverse drug events and medication errors in African hospitals: A systematic review. Drugs Real World Outcomes 2018; 5(1): 1-24.

7. British Pharmacological Society. Ten principles of good prescribing. London: British Pharmacological Society; 2010. Available from:

https://www.bps.ac.uk/BPSMemberPortal/media/BPSWebsite/Assets/BPSPrescribingStatement03Feb2010. pdf $[$ Accessed 15/11/2016].

8. World Health Organization. WHO Global Patient Safety Challenge: Medication Without Harm. NK: World Health Organization; 2017. Available from: https://www.who.int/patientsafety/medication-

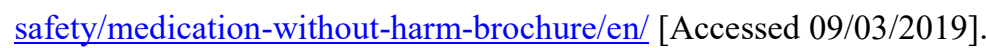

9. Jebara T, Cunningham S, Maclure K, et al. Stakeholders' views and experiences of pharmacist prescribing: A systematic review. British Journal of Clinical Pharmacology 2018; 84: 1883-1905.

10. Stewart DC, Jebara T, Cunningham S, et al. Future perspectives on nonmedical prescribing. Therapeutic Advances in Drug Safety 2017; 8(6): 183-197.

11. Department of Health and Social Security. Neighbourhood Nursing: A Focus for Care (Cumberlege Report). London: Her Majesty's Stationery Office, 1986.

12. Department of Health. Review of prescribing, supply and administration of medicines. London: UK Department of Health; 1999. Available from: 
http://webarchive.nationalarchives.gov.uk/+/www.dh.gov.uk/en/Publicationsandstatistics/Publications/Publi cationsPolicyAndGuidance/DH_4077151 [Accessed 10/04/2019].

13. Cope L, Abuzour A, Tully M. Nonmedical prescribing: where are we now? Therapeutic Advances in Drug Safety 2016; 7(4): 165-172.

14. Department of Health. Improving patient's access to medicines: A guide to implementing nurse and pharmacist independent prescribing within the NHS in England. London: UK Department of Health; 2006. Available from: http://webarchive.nationalarchives.gov.uk/+/www.dh.gov.uk/en/PublicationsandStatistics/Publications/Publ icationsPolicyandGuidance/DH_4133743 [Accessed 13/05/2016].

15. Royal Pharmaceutical Society. A competency framework for all prescribers. London: Royal Pharmaceutical Society of Great Britain; 2016. Available from:

https://www.rpharms.com/resources/frameworks/prescribers-competency-framework [Accessed 16/09/2018].

16. General Pharmaceutical Council. Standards for pharmacy education. London: General Pharmaceutical Council; 2018. Available from: https://www.pharmacyregulation.org/education/education-standards [Accessed 27/04/2018].

17. Weeks G, George J, Maclure K, Stewart D. Non-medical prescribing versus medical prescribing for acute and chronic disease management in primary and secondary care. Cochrane Database of Systematic Reviews 2016; 11: CD011227.

18. Baqir W, Crehan O, Murray R, et al. Pharmacist prescribing within a UK NHS hospital trust: Nature and extent of prescribing, and prevalence of errors. European Journal of Hospital Pharmacy 2015; 22: 79-82.

19. Lewis PJ, Dornan T, Taylor D, et al. Prevalence, incidence and nature of prescribing errors in hospital inpatients. Drug Safety 2009; 32(5): 379-389.

20. Reid F, Power A, Stewart D, et al. Piloting the United Kingdom 'Prescribing Safety Assessment' with pharmacist prescribers in Scotland. Research in Social and Administrative Pharmacy 2018; 14(1): 62-68.

21. Qatar General Secretariat for Development Planning and Statistics. Qatar National Vision 2030. Doha: Qatar General Secretariat for Development Planning and Statistics; 2008. Available from: http://www.gsdp.gov.qa/portal/page/portal/gsdp_en/qatar_national_vision [Accessed 10/01/2016].

22. Qatar Supreme Council of Health. National Health Strategy 2011-2016. Doha: Qatar Supreme Council of Health; 2013. Available from: 
http://www.qu.edu.qa/pharmacy/components/upcoming_events_material/Qatar_National_Health_Strategy.p df $[$ Accessed 10/01/2016].

23. Qatar National Research Fund. Qatar National Research Strategy (QNRS). Doha: Qatar National Research Fund; 2014. Available from: http://www.qnrf.org/en-us/About-Us/QNRS [Accessed 18/01/2016].

24. Jebara T, Cunningham S, Maclure K, et al. Key stakeholders' views on the potential implementation of pharmacist prescribing: A qualitative investigation. Research in Social and Administrative Pharmacy. In press. 2019. Available from:

https://www.sciencedirect.com/science/article/pii/S1551741119301548?via\%3Dihub [Accessed 20/06/2019].

25. Jones J, Hunter D. Qualitative research: Consensus methods for medical and health services research. British Medical Journal 1995; 311(7001):376-80.

26. Campbell S, Cantrill J. Consensus methods in prescribing research. Journal of Clinical Pharmacy and Therapeutics 2001; 26: 5-14.

27. Keeney S, Hasson F, McKenna H. The Delphi technique in nursing and health research. $1^{\text {st }}$ ed. Chichester: Wiley-Blackwell Publishing; 2011.

28. Nair R, Aggarwal R, Khanna D. Methods of formal consensus in classification/diagnostic criteria and guideline development. Seminars in Arthritis and Rheumatism 2011; 41(2): 95-105.

29. Avella JR. Delphi panels: Research design, procedures, advantages, and challenges. International Journal of Doctoral Studies 2016; 11: 305-321.

30. Delbecq AL, Van De Ven AH, Gustafson DH. Group techniques for program planning. $1^{\text {st }}$ ed. Glenview: Scott, Foresman, and Co; 1975.

31. National Association of Pharmacy Regulatory Authorities. Model standards of practice for Canadian pharmacists. Ottawa: National Association of Pharmacy Regulatory Authorities; 2009. Available from: https://napra.ca/sites/default/files/201709/Model_Standards_of_Prac_for_Cdn_Pharm_March09_layout2017_Final.pdf [Accessed 16/09/2018].

32. Centers for Disease Control and Prevention. Collaborative practice agreements and pharmacists' patient care services: A resource for pharmacists. Atlanta: US Department of Health and Human Services, Centers for Disease Control and Prevention; 2013. Available from: https://www.cdc.gov/dhdsp/pubs/docs/Translational_Tools_Pharmacists.pdf [Accessed 16/09/2018]. 
33. Pharmacy Council of New Zealand. Safe effective pharmacy practice: Standards and guidance for pharmacist prescribers. Wellington: Pharmacy Council of New Zealand; 2013. Available from: http://www.pharmacycouncil.org.nz/Portals/12/Documents/prescribers/Pharmacist_Prescriber_Standards_J uly2013.pdf?ver=2017-02-20-130511-963 [Accessed 16/09/2018].

34. Pharmaceutical Society of Northern Ireland. Standards and Guidance for Pharmacist Prescribers. Belfast: Pharmaceutical Society of Northern Ireland; 2013. Available from: http://www.psni.org.uk/wpcontent/uploads/2012/09/Standards-and-Guidance-for-Pharmacist-Prescribing-April-2013.pdf [Accessed 16/09/2018].

35. Damschroder L, Aron D, Keith R, et al. Fostering implementation of health services research findings into practice: a consolidated framework for advancing implementation science. Implementation Science 2009; 4: 50.

36. Nilsen P. Making sense of implementation theories, models and frameworks. Implementation Science 2015; 10: 53 .

37. Stewart D, Klein S. The use of theory in research. International Journal of Clinical Pharmacy 2016; 8(3): 615-619.

38. Von Der Gracht HA. Consensus measurement in Delphi studies: Review and implications for future quality assurance. Technological Forecasting \& Social Change 2012; 79: 1525-1536.

39. Bergin T. An introduction to data analysis: Quantitative, qualitative and mixed methods. $1^{\text {st }}$ ed. London: Sage Publications Ltd; 2018.

40. Glover J, Izzo D, Odato K, Wang L. EBM Pyramid. NK: Trustees of Dartmouth College and Yale University; 2006. Available from: http://www.ebmpyramid.org/images/pyramid.gif [Accessed 26/08/2018].

41. Tonna A, McCaig D, Diack L, et al. Development of consensus guidance to facilitate service redesign around pharmacist prescribing in UK hospital practice. International Journal of Clinical Pharmacy 2014; 36: 1069-1076.

42. Courtenay M, Deslandes R, Harries-Huntley G, et al. Classic e-Delphi survey to provide national consensus and establish priorities with regards to the factors that promote the implementation and continued development of non-medical prescribing within health services in Wales. BMJ Open 2018; 8(9): e024161.

43. Department of Health. Supplementary prescribing by nurses and pharmacists within the NHS in England. A guide for implementation. London: UK Department of Health; 2003. Available from: 
http://webarchive.nationalarchives.gov.uk/+/www.dh.gov.uk/en/Publicationsandstatistics/Publications/Publi cationsPolicyAndGuidance/DH_4009717 [Accessed 03/08/2016].

44. Dawoud D, Griffiths P, Maben J, et al. Pharmacist supplementary prescribing: a step toward more independence? Research in Social and Administrative Pharmacy 2011; 7(3): 246-56.

45. Cooper R, Anderson C, Avery T, et al. Nurse and pharmacist supplementary prescribing in the UK: A thematic review of the literature. Health Policy 2008; 85: 277-292.

46. Al Thani K. Law No.(9) of 1987 on Control and Regulation of Control and Regulation of Narcotic Drugs and Dangerous Psychotropic Substances (NDDPS). Doha: Qatar Legal Portal (Al-Meezan); 1987. Available from: http://www.almeezan.qa/LawPage.aspx?id=3989\&language=en [Accessed 16/09/2018].

47. NHS Education for Scotland. A Guidance for Good Prescribing Practice for Prescribing Pharmacists in NHS Scotland. Edinburgh: NHS Education for Scotland; 2012. Available from: https://www.nes.scot.nhs.uk/media/1457463/nesd0061_goodprescribingpractice.pdf [Accessed 01/11/2018].

48. Craig P, Dieppe P, Macintyre S, et al. Developing and evaluating complex interventions: New guidance. London: Medical Research Council; 2008. Available from: https://www.mrc.ac.uk/documents/pdf/complexinterventions-guidance/ [Accessed 10/04/2019]. 